\title{
REVIEW
}

\section{Laquinimod Therapy in Multiple Sclerosis: A Comprehensive Review}

\author{
Channa Kolb-Sobieraj · Sahil Gupta • Bianca Weinstock-Guttman
}

To view enhanced content go to www.neurologytherapy-open.com

Received: December 20, 2013 / Published online: May 6, 2014

(C) The Author(s) 2014. This article is published with open access at Springerlink.com

\section{ABSTRACT}

Introduction: Multiple sclerosis (MS) is considered an autoimmune disease with inflammatory and neurodegenerative underlying processes that affect the central nervous system. The available disease-modifying therapies (DMTs) approved to treat MS have only shown partial benefit in controlling the disease progression, primarily impeding its inflammatory component, while the parenteral administration of most of these therapies has shown to affect patient compliance. Laquinimod is a promising new oral

Electronic supplementary material The online version of this article (doi:10.1007/s40120-014-0017-6) contains supplementary material, which is available to authorized users.

C. Kolb-Sobieraj $(\bowtie) \cdot S$. Gupta $\cdot$ B. WeinstockGuttman $(\bowtie)$

Department of Neurology, School of Medicine and Biomedical Sciences, State University of New York at Buffalo, New York, USA

e-mail: cmkolb@buffalo.edu

B. Weinstock-Guttman

e-mail: bw8@buffalo.edu;

BWeinstock-Guttman@kaleidahealth.org

C. Kolb-Sobieraj · S. Gupta · B. Weinstock-Guttman Baird MS Center, Jacobs Neurological Institute, Buffalo General Medical Center, New York, USA

S. Gupta

e-mail: sahil.mamc@gmail.com drug recently evaluated in a third phase III clinical trial that demonstrated beneficial effects in delaying disease progression and preventing brain atrophy, suggesting a potential neuroprotective effect and a favorable safety profile.

Areas Covered: This is a comprehensive review covering clinical efficacy and safety data obtained from two phase III clinical trials, as well as the presumed beneficial mechanism of action, of laquinimod. This article also provides a short overview of the oral DMTs recently approved for the treatment of relapsing MS, as well as challenges that still remain to be overcome to fully control the relentless course of MS.

Conclusion: Laquinimod has been shown to have a novel immunomodulatory and potential neuroprotective mechanism of action as suggested from animal models and in vitro experimental data. Phase III clinical trials ALLEGRO (Clinicaltrials.gov \#NCT00509145) and BRAVO (Clinicaltrials.gov \#NCT00605215) have demonstrated clinical efficacy and tolerability, while the third phase III study is currently evaluating the safety and efficacy of laquinimod at a higher dosage. Emerging oral treatments like laquinimod will provide new options for patients to consider that can lead to 
better patient adherence and improved outcomes.

Keywords: Clinical trials; Disease modifying therapies; Laquinimod; Multiple sclerosis; Neurology; Neuroprotection

\section{INTRODUCTION}

New oral disease-modifying therapies (DMTs) are currently available for the treatment of relapsing multiple sclerosis (MS). Laquinimod (quinolin-3carboxamide) is one of the new once-a-day oral medications being studied as a potential treatment option for relapsing remitting MS [1]. Laquinimod is structurally similar to another compound, roquinimex (linomide) which was also studied as a potential treatment for MS [2]. Roquinimex was found in early phase II and III trials to prevent clinical relapses and magnetic resonance imaging (MRI) activity in MS. However, the phase III trials were halted due to unexpected serious adverse events such as pericarditis, serositis and myocardial infarction that were not detected during the phase II trials $[3,4]$. Following the failed clinical trial with roquinimex, laquinimod was discovered by Jonsson et al. [5] in a structure activity screening program to find compounds with efficacy against autoimmune disorders but lacking the side effects of roquinimex. Chemical modifications involving the quinolone ring and elongation of the amidic methyl group were performed on the roquinimex structure which led to the discovery of laquinimod with the chemical structure $N$-ethyl- $N$-phenyl-5chloro-1, 2-dihydro-4-hydroxy-1-methyl-2-oxo-3quinoline-carboxamide. These modifications led to a 20 -fold increase in potency of laquinimod in treating animal models of experimental autoimmune encephalomyelitis (EAE) with a relatively favorable safety profile [6].
MS is considered an autoimmune disease with inflammatory and neurodegenerative underlying processes that affect the central nervous system (CNS). Auto-reactive $\mathrm{T}$ lymphocytes, B cells and macrophages enter the CNS causing myelin and axonal destruction and further chronic microglial activation [7]. Axonal damage and microglial activation lead to the irreversible and relentless clinical progression in patients with MS [8]. Due to the heterogeneous response to therapies between patients, often with suboptimal efficacy, and concern for side effects associated with current DMTs, new therapies are sought to address these concerns. In particular, patients with progressive MS have a great unmet need for effective interventions. Developing new therapies such as laquinimod may help address some of these deficiencies.

This review is based on previously conducted studies and does not involve any new studies of human or animal subjects performed by any of the authors.

\section{NOVEL MECHANISM OF ACTION}

Laquinimod has shown to exert beneficial effects on many in vitro and in vivo models of MS. A graphic synopsis of its potential mechanism of action is represented in Fig. 1. Laquinimod was found to influence the ratio of pro-inflammatory versus anti-inflammatory cytokine production in EAE rat models and peripheral blood mononuclear cells from healthy volunteers [9]. Cytokine analysis of EAE-induced rats treated with laquinimod revealed a decrease in proinflammatory markers, tumor necrosis factor- $\alpha$ and interleukin (IL)-12. Concurrently, an increase in anti-inflammatory markers transforming growth factor- $\beta$ and IL- 4 were noted in the same laquinimod-treated EAE rats [9]. It was also found 


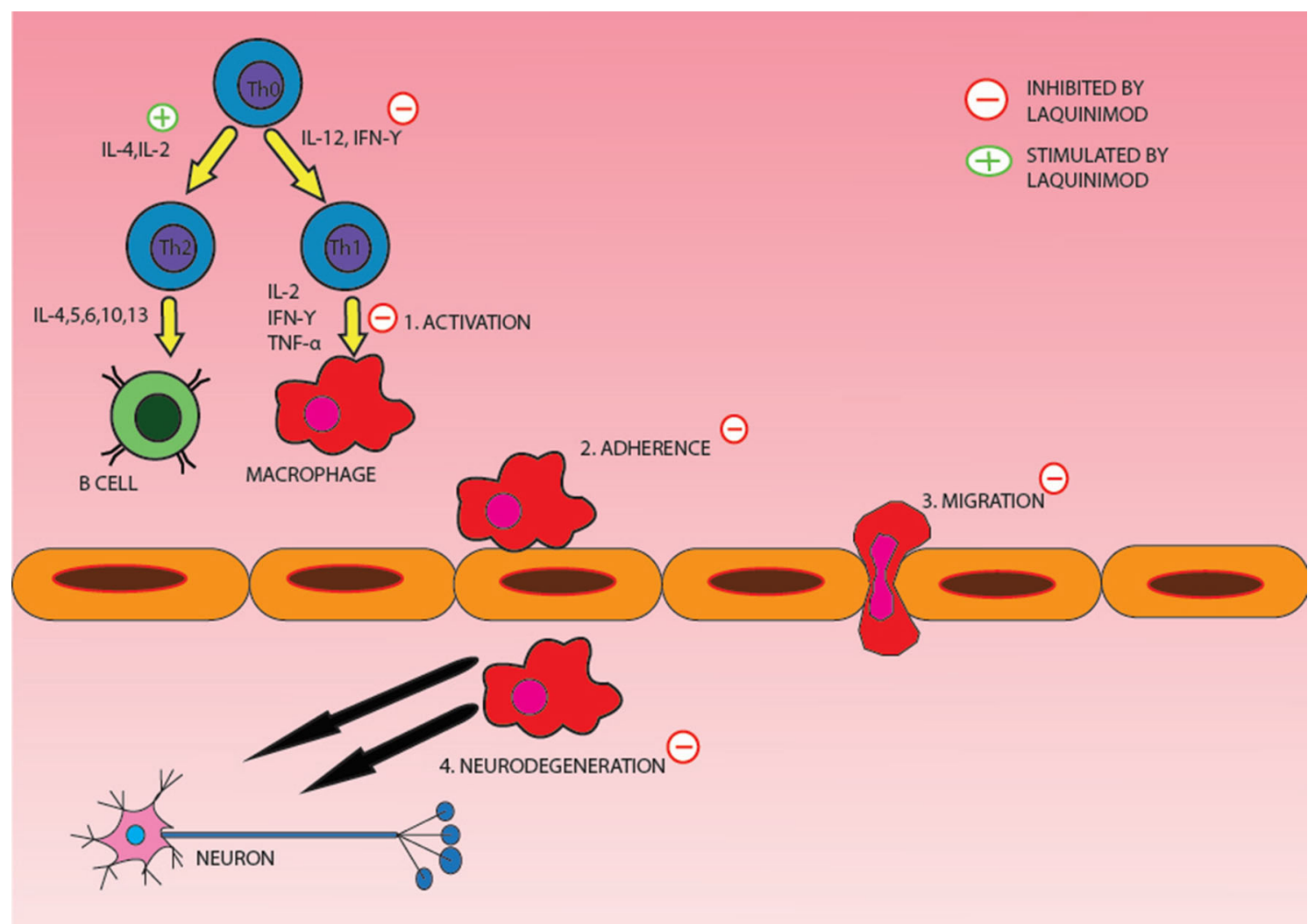

Fig. 1 The figure illustrates the mechanism of action of laquinimod and its presumed site of action. $I F N$ interferon, $I L$ interleukin, TNF tumor necrosis factor

that laquinimod given preventatively blocked the entry of inflammatory $\mathrm{T}$ cells into the CNS. Preventative treatment with laquinimod also delayed clinical presentation of EAE in a dosedependent manner [10]. A dose-dependent effect of laquinimod on reducing another proinflammatory cytokine, IL-17, was also seen in another experiment involving EAE rats treated with laquinimod versus placebo [11]. The same team observed a decrease in IL-17 and other proinflammatory markers, IL-3 and granulocyte colony-stimulating factor in peripheral blood mononuclear cells from healthy human controls [9].

Laquinimod was also found to inhibit acute EAE clinical symptoms when administered after the mice developed clinical symptoms of progressive paresis. Oral laquinimod was able to suppress clinical signs of disease in doses of $1 \mathrm{mg} / \mathrm{kg} /$ day and higher [6]. Laquinimod, given at the time of symptom onset in myelin oligodendrocyte glycoprotein-induced EAE, reduced the severity of the disease at the dose of $25 \mathrm{mg} / \mathrm{kg} /$ day. This was considered the therapeutic dose. In another experiment, laquinimod was given preventively at the time of disease induction at doses of $5 \mathrm{mg} / \mathrm{kg}$ or 25 $\mathrm{mg} / \mathrm{kg} /$ day. It was found that laquinimod had a dose-dependent effect on preventing clinical disease presentation of EAE in EAE-induced mice [11].

Laquinimod was found to act more like an immune modulating drug, rather than an immunosuppressive one. Laquinimod-treated rats were able to mount a cellular and humoral response, including humoral 
immunoglobulin (Ig)M and IgG against a presented pathogen [12]. Laquinimod did not affect the proliferation or survival of peripheral blood mononuclear cells in healthy human subjects exposed to different concentrations of the drug [12]. The viability of cardiac allograft tissue in the presence of laquinimod also testifies to its lack of immunosuppressive effect [9].

Oral laquinimod penetrates the CNS in physiologic and inflammatory pathologic conditions. When oral ${ }^{14} \mathrm{C}$-laquinimod was administered to track its distribution to the brain and spinal cord in EAE and healthy mice, healthy rats showed a 7-8\% laquinimod distribution to the brain and spinal cord in relation to the peripheral blood concentration at $2 \mathrm{~h}$ post dose, while EAE rats revealed a 13\% distribution of laquinimod to the brain and spinal cord in relation to the peripheral blood at an hour post dose [9]. A parallel time to the peak concentration of laquinimod in the peripheral blood and the CNS was identified in the EAE model [9].

Laquinimod is thought to play a role in neuroprotection as evidenced by its influence on brain-derived neurotrophic factor (BDNF) levels. BDNF is a protein that is involved in strengthening synapses and promoting growth and differentiation of new neurons. It is also an important factor in promoting survival of neurons in the central and peripheral nervous system [13]. As part of a phase II trial, patients $(N=200)$ given laquinimod $0.6 \mathrm{mg} /$ day versus placebo had their serum BDNF levels checked at baseline, at 3 months and then at 9 months. It was found that the laquinimod patients had significantly higher levels of serum BDNF by month three compared to placebo $(P<0.01)$ [14].

Axonal protection was also seen in animal models treated with laquinimod. In one experiment, rats were treated preventively (the day of EAE inoculation) with laquinimod to measure the degree of axonal damage in a model of optic neuritis [6]. Axonal damage and loss were measured by the accumulation of amyloid precursor protein antibodies at the sites of axonal damage and by the number of fiber counts within the optic nerve. It was observed that the day of disease manifestation was significantly delayed in the group which received laquinimod in the dosage of $5 \mathrm{mg} / \mathrm{kg}$ subcutaneously. However, the severity of symptoms did not differ between the groups, which leads us to believe that laquinimod effectively delays disease onset autoimmune optic neuritis [15]. Another study used scanning electron microscopy to visualize axonal damage in the spinal cords of EAE mice treated preventively with laquinimod versus placebo [6]. The spinal cords of the laquinimod-treated rats showed less demyelination, inflammation and axonal loss compared to the controls [6].

\section{CLINICAL STUDIES WITH LAQUINIMOD}

Various phase I trials were performed to assess the safety and tolerability of laquinimod. Eight phase I trials used laquinimod with doses ranging from 0.1 to $2.4 \mathrm{mg} /$ day and found it to be well tolerated [16]. The doses of 0.1 and $0.3 \mathrm{mg} /$ day were subsequently used in the phase IItrials.

Two phase II trials and one phase IIb trial extension have been conducted to evaluate the efficacy, safety and tolerability of laquinimod compared to placebo [17-19]. The phase II study conducted by Polman et al. [17] was a 24-week, multicenter, double blind and three-armed randomized trial. The primary outcome 
measure was the cumulative number of active lesions over the length of the study. Patients ( $N=209)$ were randomized into three groups: Laquinimod $0.3 \mathrm{mg} /$ day, laquinimod $0.1 \mathrm{mg} /$ day and placebo. The primary outcome was defined as the mean cumulative number of active MRI lesions. Patients had MRI scans at baseline, weeks 4,8 and 24, and 8 weeks post discontinuation of therapy. The mean cumulative number of active lesions decreased by $44 \%$ in the group treated with $0.3 \mathrm{mg} /$ day of laquinimod when compared to placebo $(P=0.0498)$ [17]. The safety and tolerability profile were found to be good and the adverse events were similar in the placebo versus the treatment groups. The investigators observed a small increase in the erythrocyte sedimentation rate and abnormal liver function test in the treatment group but it was clinically insignificant and did not warrant treatment discontinuation [17].

Another phase IIb trial (LAQ/5062; Clinicaltrials.gov \#NCT00349193) compared two doses of laquinimod ( 0.3 and $0.6 \mathrm{mg} /$ day) with placebo over a period of 36 weeks [19]. Three hundred and eight patients with active MS disease were randomized and assigned to one of the three groups. The primary outcome measure was the cumulative number of gadolinium-enhancing lesions at weeks 24, 28, 32 and 36 (last 4 scans of the treatment period) [19]. The investigators observed a $40.4 \%$ reduction in cumulative number of gadolinium-enhancing lesions and 44\% reduction in the cumulative number of new T2 lesions for $0.6 \mathrm{mg} /$ day laquinimod as compared to placebo. Annualized relapse rate (ARR) also decreased in the $0.6 \mathrm{mg} /$ day treatment group by $32 \%$ but did not reach statistical significance. Surprisingly, the group treated with $0.3 \mathrm{mg} /$ day dose of laquinimod showed no significant differences from the placebo group [19]. This was unforeseen as the results from Polman et al. [17] suggested promising results from the $0.3 \mathrm{mg} /$ day laquinimod-treatment group. This difference in findings was attributed to the increased sensitivity of the triple dose gadolinium used in the trial by Polman et al. [17] and was also believed that the lower dose of laquinimod took longer time to reach statistical significance [19].

After the completion of LAQ/5062 trial, 257 out of the 308 subjects were enrolled in a double-blind extension study (LAQ/5063; Clinicaltrials.gov \#NCT00745615) for another 36 weeks [18]. The treatment arm subjects were continued on the medication but the subjects on placebo arm were switched to laquinimod 0.3 or $0.6 \mathrm{mg} /$ day. Among the patients switched from placebo group to the treatment group, there was a $52 \%$ decrease $(P=0.0006)$ in the mean number of gadolinium-enhancing lesions between the start and the end of the extension phase. The reduction was more significant in subjects that switched to laquinimod $0.6 \mathrm{mg} /$ day $(P<0.009)$ group in comparison to laquinimod $0.3 \mathrm{mg} /$ day group $(P<0.03)$. Subjects that continued on the treatment group showed sustained benefit throughout the extension [18]. The safety and tolerability profile was good in both LAQ/5062 trial and its extension. A few serious adverse events occurred in the treatment group in LAQ/5062 trial which could potentially be attributed to laquinimod. One case of Budd-Chiari syndrome was found in the laquinimod $(0.6 \mathrm{mg} /$ day $)$ group. The patient was taken off of laquinimod but was later found to be heterozygous for Factor $\mathrm{V}$ Leiden mutation [18]. Another patient in the laquinimod ( $0.3 \mathrm{mg} /$ day) group developed marked increase in liver function tests. Once the drug was discontinued, the liver function tests gradually normalized. Herpetic infections appeared more 
frequently in the $0.3 \mathrm{mg} /$ day treatment group but the difference in overall infection rates did not differ between the treatment and the placebo group. Safety and tolerability remained unchanged during the extension study and no unexpected adverse events emerged except for three patients who discontinued laquinimod due to liver enzyme elevations [18]. Several patients in the treatment group developed arthralgia but recovered without drug discontinuation. Surprisingly, the adverse effects due to roquinimex, like pleuritis, pericarditis and myocardial infarction, were absent in the patients treated with laquinimod in the clinical trials.

Following the encouraging phase II results, the sponsors designed the phase III trials with laquinimod at $0.6 \mathrm{mg} /$ day dosage. ALLEGRO (Assessment of oral Laquinimod in preventing progression in Multiple Sclerosis; Clinicaltrials.gov \#NCT00509145) was a multicenter, randomized controlled phase III trial in which 1,106 patients were randomly assigned to placebo or laquinimod $(0.6 \mathrm{mg} /$ day $)$ in 1:1 ratio and followed up for 24 months [1]. The primary end point was the ARR at 24 months. Key secondary end points included the total number of gadolinium-enhancing lesions and new or enlarging T2-weighted lesions on MRI. The reduction in ARR for the treatment group, although reaching significance, was modest as compared to the placebo group $(0.30$ vs. 0.39 , respectively; $P=0.002)$. Gadolinium-enhancing lesions (1.33 vs. 2.12 , respectively; $P<0.001$ ) and the new/enlarging T2 lesions on MRI (5.03 vs. 7.14, respectively; $P<0.001$ ) were also decreased in the treatment group as compared to the placebo group. Laquinimod was found to be well tolerated in that $11.1 \%$ of patients suffered serious adverse events while on laquinimod as compared to $9.5 \%$ of patients on placebo [1]. Most common adverse events seen more frequently in laquinimod treatment as compared to placebo were elevated liver enzymes $(30 \%$ vs. $17.7 \%$, respectively), headache (22.7\% vs. $17.8 \%$, respectively), back pain (16.4\% vs. $9.0 \%$, respectively), arthralgia (8.5\% vs. $7.6 \%$, respectively) and diarrhea $(8.0 \%$ vs. $6.1 \%$ ). All cases of elevated liver enzymes were reversible either with treatment continuation or within 2 months of treatment discontinuation. Urinary tract infection $(7.3 \%$ vs. $4.5 \%$, respectively) and sinusitis (5.3\% vs. $4.5 \%$, respectively) occurred more in laquinimod groups as compared to the placebo groups but no opportunistic infections were identified in either group [1].

BRAVO (Benefit-risk assessment of Avonex and Laquinimod; Clinicaltrials.gov \#NCT00605215) was a second phase III clinical trial aimed to assess the efficacy, safety and tolerability of laquinimod in comparison to interferon (IFN) and placebo [20]. More than 1,300 patients were randomized $1: 1: 1$ to oral laquinimod, IFN- $\beta 1 \mathrm{~A}$ and placebo for 24 months. The primary endpoint was the ARR which did not reach statistical significance, but showed a trend to reduction with laquinimod [Risk ratio $(\mathrm{RR})=0.823$, 95\% CI 0.664-1.020; $P=0.075]$. However, following an adjustment for imbalance of baseline MRI disease activity between groups, the analysis demonstrated that laquinimod significantly reduced ARR compared to placebo $(0.29$ vs. 0.37 , $\mathrm{RR}=0.787, \quad 95 \% \mathrm{CI}=0.637-0.972 ; \quad P=0.026)$, reduction in risk of disability progression (Hazard ratio $=0.665 ; P=0.44$ ) and brain atrophy on MRI (27.5\%; $P<0.0001)$ [20]. Safety profile in BRAVO was found to be similar to that of ALLEGRO. Following a similar adjusted analysis, ARR was found to be reduced in the IFN $\beta$ group (0.27) compared to 
placebo $(0.29 ; P=0.002)$. Disability progression was reduced in IFN as compared to placebo (28.7\%; $P=0.089)$ but no treatment effect of IFN was observed on brain atrophy [20].

A third phase III trial, CONCERTO (Clinicaltrials.gov \#NCT01707992), is currently ongoing and aims to evaluate two doses of laquinimod $(0.6$ and $1.2 \mathrm{mg} /$ day $)$ in approximately 1,800 patients for 24 months. The primary outcome measure will be disability progression measured by the Expanded Disability Status Scale (EDSS) [21].

\section{CURRENT TREATMENT AND UNMET NEED}

The number of DMTs available today for treating relapsing MS increased significantly during the past decade and is expected to increase further [22]. Glatiramer acetate and IFN are still the drugs currently used as first-line treatment in most countries, including the USA [23-25]. These drugs are administered parenterally and have favorable long-term safety profiles. However, less than half of patients respond to first-line therapies and the adherence is often limited because of their parenteral use including injection site reactions. Other DMTs, like natalizumab or the Food and Drug Administration (FDA) approved oral drugs, can be considered [26]. Fingolimod, teriflunomide and dimethyl fumarate are the oral treatments which have been approved for the treatment of MS (Table 1) [1, 20, 27-35]. Many more oral drugs, including laquinimod, are on the horizon and are in different phases of their respective clinical trials.

Despite the significant advances in the field of MS, some glaring unmet needs still remain. One of the most important challenges yet to be addressed is whether the current treatments hold the potential for completely arresting the MS disease process. With the advent of drugs like natalizumab and alemtuzumab, this notion has emerged, especially if administered early in the course of the disease, but further research is still required. The benefit seen with natalizumab, despite its high efficacy on controlling the inflammatory process, is hampered by the risk of developing progressive multifocal leukoencephalopathy, a potentially fatal and devastating disease, while alemtuzumab is associated with a high risk of developing other autoimmune diseases [33].

Another challenge that will require further investigation is the lack of specific biomarkers that can help select the best therapy for the individual patient. As new therapies with different mechanisms of action become available, the possibility of developing personalized therapy based on disease severity and patient characteristics is a challenging but actively pursued process.

\section{HOW IT WILL FIT INTO THE CURRENT TREATMENT REGIMENS?}

The upcoming oral drugs not only provide convenient options for treatment of MS but also pose a plethora of new challenges. The severity of the disease and the risk-benefit profile of the drugs would be instrumental in determining the treatment strategies for individual patients and constructing a personalized management plan.

Oral drugs provide a more convenient and suitable option for patients and are likely to be prescribed more regularly as soon as their longterm safety profiles are determined. Laquinimod features as an attractive option for treatment of MS because of its very favorable safety profile 
Table 1 Comparison of results of phase III clinical trials of different oral therapies for RRMS

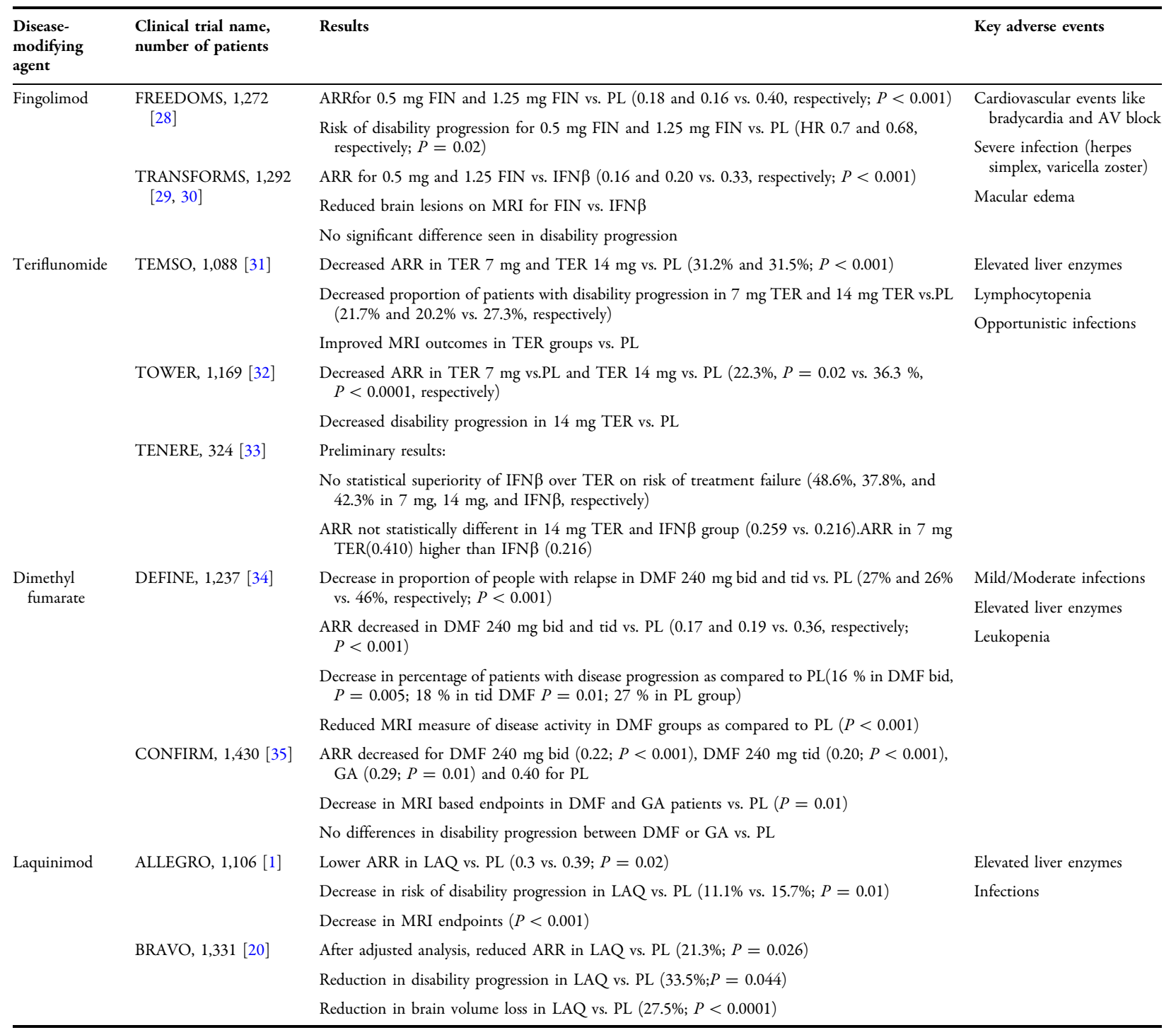

Clinical trial acronyms: ALLEGRO, Placebo Controlled Trial of Oral Laquinimod for Multiple Sclerosis; BRAVO, Laquinimod Double Blind Placebo Controlled Study in RRMS Patients With a Rater Blinded Reference Arm of Interferon $\beta$-1a; CONFIRM, Comparator and an Oral Fumarate in RRMS; DEFINE, Determination of the Efficacy and Safety of Oral Fumarate in RRMS; FREEDOMS, A placebo-controlled trial of oral Fingolimod in Relapsing Multiple Sclerosis; TEMSO, The Teriflunomide Multiple Sclerosis Oral Trial; TENERE, A study comparing the effectiveness and safety of Teriflunomide and Interferon Beta-la in Patients with Relapsing multiple sclerosis; TOWER, Teriflunomide efficacy and safety in patients with relapsing multiple sclerosis; TRANSFORMS, Trial Assessing Injectable Interferon versus FTY720 Oral in Relapsing-Remitting Multiple Sclerosis

$A R R$ annualized relapse rate, $A V$ atrioventricular, bid twice a day, $D M F$ dimethyl fumarate, FIN fingolimod, GA Glatirameracetate, HR Hazard ratio, IFN interferon, $L A Q$ laquinimod, $M R I$ magnetic resonance imaging, $P L$ placebo, TER teriflunomide, tid three times a day

and tolerability [1]. The beneficial effect associated with the use of laquinimod on preventing disability progression and brain atrophy as seen from the clinical trials may be an attractive option for patients with a milder inflammatory disease course [36]. Due to its higher disability reduction rates, laquinimod can be an attractive option for patients with higher EDSS scores [1]. The higher dose data $(1.2 \mathrm{mg} /$ day), presently under investigation, will be very important in defining the specific group of patients that will best benefit from this medication [21]. Laquinimod can be also prescribed to patients who have discontinued 
the use of the parenteral first-line agents due to side effects [27].

A few shortcomings of laquinimod should also be considered while prescribing the drug. First, $30 \%$ of the patients treated with laquinimod in the ALLEGRO clinical trial suffered from elevated liver enzymes. Therefore, continuous monitoring of liver enzymes is advised while prescribing laquinimod [1]. Second, although laquinimod maybe a safer drug, it is less effective as compared to the already approved oral DMTs and, therefore, shall be considered as an add-on therapy to other DMTs [37]. Further studies are also required to establish the efficacy of laquinimod in progressive forms of MS disease like primary progressive MS and secondary progressive MS.

\section{CONCLUSION}

Laquinimod is a new oral medication evaluated for the treatment of relapsing MS patients which appears to be a convenient and suitable option for patients to consider. Its favorable safety profile and tolerability differentiates it from the other emerging therapies. Elevated liver enzymes is the only concerning side effect of laquinimod which has been observed. This side effect has proved to be reversible within 2 months of treatment discontinuation $[16,18]$. Laquinimod acts through a multipronged immunomodulatory mechanism of action and may also exert a neuroprotective effect on the neurons. Histopathological analysis of the EAE model shows that laquinimod acts via decreasing the infiltration of CD4+ cells and macrophages into CNS and neuroprotection through preventing axonal damage $[9,11]$. This multifaceted effect makes it an attractive treatment option. Clinical trials, like ALLEGRO and BRAVO, have established laquinimod as a safe DMT, but lesser effective than the already FDA-approved DMTs [1, 18, 20]. Therefore, laquinimod can be considered as an add-on drug to other MS therapies. If the ongoing phase III trials confirm its efficacy and safety, laquinimod would be a welcome and a muchneeded addition to the therapeutic options for treatment of patients with relapsing and possibly progressive MS [21]. All in all, the impending arrival of the new oral therapies will welcome a paradigm shift in the treatment of relapsing MS with convenient drug administration, better patient compliance and better outcome.

\section{ACKNOWLEDGMENTS}

During the peer review process, the manufacturer of the agent under review was offered an opportunity to comment on the article. No comments or subsequent changes were made as a result of this. No funding or sponsorship was received for this study or publication of this article. All named authors meet the ICMJE criteria for authorship for this manuscript, take responsibility for the integrity of the work as a whole and have given final approval for the version to be published.

Conflict of interest. Dr. Channa Kolb has participated in speaker's bureaus and has served as a consultant for Biogen Idec, Teva Neurosciences, EMD Serono, and Novartis. Dr. Sahil Gupta has no conflict of interest to disclose. Dr. Bianca Weinstock-Guttman has participated in speaker's bureaus and served as a consultant for Biogen Idec, Teva Neurosciences, EMD Serono, Pfizer, Novartis, Genzyme \& Sanofi, Mylan, and Acorda. She also has received grant/research support from the 
agencies listed above as well as ITN, Questcor and Shire. No other industry financial relationships exist.

Compliance with ethics guidelines. This review is based on previously conducted studies and does not involve any new studies of human or animal subjects performed by any of the authors.

Open Access. This article is distributed under the terms of the Creative Commons Attribution Noncommercial License which permits any noncommercial use, distribution, and reproduction in any medium, provided the original author(s) and the source are credited.

\section{REFERENCES}

1. Comi G, Jeffery D, Kappos L, et al. Placebocontrolled trial of oral laquinimod for multiple sclerosis. N Engl J Med. 2012;366:1000-9.

2. Noseworthy JH, Wolinsky JS, Lublin FD, et al. Linomide in relapsing and secondary progressive MS: part I: trial design and clinical results. North American Linomide Investigators. Neurology. 2000;54:1726-33.

3. Wolinsky JS, Narayana PA, Noseworthy JH, et al. Linomide in relapsing and secondary progressive Ms: part II: MRI results. MRI Analysis Center of the University of Texas-Houston, Health Science Center, and the North American Linomide Investigators. Neurology. 2000;54:1734-41.

4. Tan IL, Lycklama a Nijeholt GJ, Polman CH, Ader HJ, Barkhof F. Linomide in the treatment of multiple sclerosis: MRI results from prematurely terminated phase-III trials. Mult Scler. 2000;6:99-104.

5. Jonsson S, Andersson G, Fex T, et al. Synthesis and biological evaluation of new 1,2-Dihydro-4Hydroxy-2-Oxo-3-Quinolinecarboxamides for treatment of autoimmune disorders: structureactivity relationship. J Med Chem. 2004;47:2075-88.

6. Brunmark C, Runstrom A, Ohlsson L, et al. The new orally active immuno regulator laquinimod (Abr215062) effectively inhibits development and relapses of experimental autoimmune encephalomyelitis. J Neuroimmunol. 2002;130:163-72.
7. Steinman L. Multiple sclerosis: a coordinated immunological attack against myelin in the central nervous system. Cell. 1996;85:299-302.

8. Prat A, Antel J. Pathogenesis of multiple sclerosis. Curr Opin Neurol. 2005;18:225-30.

9. Brück W, Wegner C. Insight into the mechanism of laquinimod action. J Neurol Sci. 2011;306:173-9.

10. Yang JS, Xu LY, Xiao BG, Hedlund G, Link H. Laquinimod (Abr-215062) suppresses the development of experimental autoimmune encephalomyelitis, modulates the Th1/Th2 balance and induces the Th3 Cytokine Tgf-Beta in Lewis Rats. J Neuroimmunol. 2004;156:3-9.

11. Wegner C, Stadelmann C, Pförtner R, et al. Laquinimod interferes with migratory capacity of $\mathrm{T}$ cells and reduces IL-17 levels, inflammatory demyelination and acute axonal damage in mice with experimental autoimmune encephalomyelitis. J Neuroimmunol. 2010;227:133-43.

12. Gurevich M, Gritzman T, Orbach R, Tuller T, Feldman A, Achiron A. Laquinimod suppress antigen presentation in relapsing-remitting multiple sclerosis: in-vitro high-throughput gene expression study. J Neuroimmunol. 2010;221:87-94.

13. Huang EJ, Reichardt LF. Neurotrophins: roles in neuronal development and function. Annu Rev Neurosci. 2001;24:677-736.

14. Thöne J, Ellrichmann G, Seubert $\mathrm{S}$, et al. Modulation of autoimmune demyelination by laquinimod via induction of brain-derived neurotrophic factor. Am J Pathol. 2012;180:267-74.

15. Suhs K-W. Effects of laquinimod on axon degeneration in autoimmune optic neuritis. Mult Scler 2007;13:S7-S273.

16. Thone J, Gold R. Laquinimod: a promising oral medication for the treatment of relapsing-remitting multiple sclerosis. Expert Opin Drug Metab Toxicol. 2011;7:365-70.

17. Polman C, Barkhof F, Sandberg-Wollheim M, et al. Treatment with laquinimod reduces development of active MRI lesions in relapsing MS. Neurology. 2005;64:987-91.

18. Comi G, Abramsky O, Arbizu T, et al. Oral laquinimod in patients with relapsing-remitting multiple sclerosis: 36 -week double-blind active extension of the multi-centre, randomized, double-blind, parallel-group placebo-controlled study. Mult Scler. 2010;16:1360-6.

19. Comi G, Pulizzi A, Rovaris M, et al. Effect of laquinimod on MRI-monitored disease activity in 
patients with relapsing-remitting multiple sclerosis: a multicentre, randomised, double-blind, placebocontrolled phase IIb study. Lancet. 2008;371:2085-92.

20. Vollmer T, Soelberg Sorensen P, Arnold DL, on behalf of the BRAVO study group. A placebocontrolled and active comparator phase III trial (Bravo) for relapsing-remitting multiple sclerosis. Amsterdam, Netherlands: 5th joint triennial congress of the European and Americas Committees for Treatment and Research in Multiple Sclerosis; 2011.

21. Clinicaltrials.gov. The efficacy and safety and tolerability of laquinimod in subjects with relapsing remitting multiple sclerosis (RRMS) (CONCERTO). http://clinicaltrials.gov/show/ NCT01707992 (last accessed April 9, 2014).

22. Waubant E. Overview of treatment options in multiple sclerosis. J Clin Psychiatry. 2012;73:e22.

23. La Mantia L, Munari LM, Lovati R. Glatiramer acetate for multiple sclerosis. Cochrane Database Syst Rev. 2010;CD004678.

24. Jacobs LD, Cookfair DL, Rudick RA, et al. Intramuscular interferon beta-1a for disease progression in relapsing multiple sclerosis. The multiple sclerosis collaborative research group (MSCRG)'. Ann Neurol. 1996;39:285-94.

25. IFNB Multiple Sclerosis Study Group. Interferon beta- $1 \mathrm{~b}$ is effective in relapsing-remitting multiple sclerosis I. Clinical results of a multicenter, randomized, double-blind, placebo-controlled trial. Neurology. 1993;43:655.

26. Thone J, Ellrichmann G. Oral available agents in the treatment of relapsing remitting multiple sclerosis: an overview of merits and culprits. Drug Healthc Patient Saf. 2013;5:37-47.

27. Killestein J, Rudick RA, Polman CH. Oral treatment for multiple sclerosis. Lancet Neurol. 2011;10:1026-34.

28. Kappos L, Radue EW, O'Connor P, et al. A placebocontrolled trial of oral fingolimod in relapsing multiple sclerosis. N Engl J Med. 2010;362:387-401.

29. Cohen JA, Barkhof F, Comi G, et al. Fingolimod versus intramuscular interferon in patient subgroups from transforms. J Neurol. 2013;260:2023-32.

30. Doggrell SA. Oral fingolimod for relapsingremitting multiple sclerosis Evaluation of: Kappos $\mathrm{L}$, Radue E-M, O'Connor $\mathrm{P}$, et al. A placebocontrolled trial of oral fingolimod in relapsing multiple sclerosis. N Engl J Med 2010;362:387-401; and Cohen JA, Barkhof F, Comi $G$, et al. Oral fingolimod or intramuscular interferon for relapsing multiple sclerosis. N Engl J Med 2010;362:402-15. Expert Opin Pharmacother. 2010;11(10):1777-81.

31. O'Connor P, Wolinsky JS, Confavreux C, et al. Randomized trial of oral teriflunomide for relapsing multiple sclerosis. N Engl J Med. 2011;365:1293-303.

32. Kappos L, Comi G, Confavreux C. The efficacy and safety of teriflunomide in patients with relapsing MS: results from tower, a phase III, placebocontrolled study. Lyon, France: 28th Congress of the European committee for research and treatment in multiple sclerosis (ECTRIMS); 2012.

33. Clinicaltrials.gov. A study comparing the effectiveness and safety of teriflunomide and interferon beta-1a in patients with relapsing multiple sclerosis (TENERE). http://clinicaltrials. gov/show/NCT00883337 (last accessed April 9, 2014).

34. Bar-Or A, Gold R, Kappos L, et al. Clinical efficacy of BG-12 (Dimethyl Fumarate) in patients with relapsing-remitting multiple sclerosis: subgroup analyses of the define study. J Neurol. 2013;260:2297-305.

35. Hutchinson M, Fox RJ, Miller DH, et al. Clinical efficacy of BG-12 (Dimethyl Fumarate) in patients with relapsing-remitting multiple sclerosis: subgroup analyses of the confirm study. J Neurol. 2013;260:2286-96.

36. Barkhof F, Calabresi P, Miller D, Reingold S. Imaging outcomes for neuroprotection and repair in multiple sclerosis trials. Nat Rev Neurol. 2009;5(256-266):39.

37. Rammohan K, Shoemaker J. Emerging multiple sclerosis oral therapies. Neurology. 2010;74:s47-53. 